\title{
HOTAIR is a promising novel biomarker in patients with thyroid cancer
}

\author{
YIFEI ZHANG $^{1 *}$, SUI YU ${ }^{2 *}$, LIXIN JIANG $^{1}$, XIXUN WANG ${ }^{1}$ and XIAOJING SONG ${ }^{1}$ \\ Departments of ${ }^{1}$ Thyroid and Gastrointestinal Surgery, and ${ }^{2}$ Endocrinology; \\ The Affiliated Yantai Yuhuangding Hospital of Qingdao University Medical College, \\ Yantai, Shandong 264000, P.R. China
}

Received June 2, 2015; Accepted September 6, 2016

DOI: $10.3892 /$ etm.2017.4231

\begin{abstract}
Thyroid cancer (TC) is the most common endocrine malignancy. Lack of effective early diagnostic tools is one of the clinical obstacles for TC treatment. Thus, enhanced comprehension of the molecular changes in TC tumorigenesis is urgently needed to develop novel strategies for the diagnosis and treatment of TC. Long non-coding RNAs (lncRNAs) manage fundamental biochemical and cellular processes in tumorigenesis and development. One of the best-described lncRNAs, HOX transcript antisense RNA (HOTAIR), functions as a regulatory molecule in a wide variety of biological processes, and represses gene expression through recruitment of the chromatin modifying complex. However, the function of HOTAIR in TC remains unclear. In the current study, the expression of HOTAIR is elevated in TC and correlates with metastasis and poor prognosis. Furthermore, the expression of HOTAIR is significantly upregulated in human thyroid carcinoma cells compared with normal human thyroid cells. Furthermore, knockdown of HOTAIR significantly inhibited cell growth and invasion in TPC-1 and SW579 human thyroid carcinoma. In summary, HOTAIR is a promising novel biomarker in patients with TC.
\end{abstract}

\section{Introduction}

Thyroid carcinoma (TC) is the most common endocrine malignancy accounting for $>90 \%$ of endocrine gland malignancies (1). Effective treatment in the majority of patients with TC includes total thyroidectomy followed by therapy with

Correspondence to: Dr Xiaojing Song, Department of Thyroid and Gastrointestinal Surgery, The Affiliated Yantai Yuhuangding Hospital of Qingdao University Medical College, 20 Yuhuangding Road, Zhifu, Yantai, Shandong 264000, P.R. China

E-mail: songxiaojing_0429@163.com

*Contributed equally

Key words: long non-coding RNAs, HOX transcript antisense RNA, thyroid cancer, biomarker radioactive iodide (2). Although the prognosis of patients with TC is favorable, unfortunately, the capacity for radioactive iodide accumulation is diminished or lost in a high proportion of patients with metastases (3). The genetic events involved in TC consist of numerous gene mutations and gene dysregulation, and several signaling pathways are activated in favor of cell growth, survival and angiogenesis (4). However, our understanding of the detailed molecular mechanism underlying TC tumorigenesis and development is still not clear.

Accumulating evidence has demonstrated that noncoding RNAs are involved in various types of human cancers (5). To date, numerous long noncoding RNAs (lncRNAs) have been demonstrated to have important roles in human normal or disease states. Homeobox transcript antisense RNA (HOTAIR) is a 2,000 bp IncRNA that is encoded antisense to the HOXC locus (6). HOTAIR regulates the transcriptional silencing of genes by binding to polycomb repressive complex 2 (PRC2) and localizing to the specific site where H3K27 trimethylation and epigenetic silencing of gene expression occur (7). In addition, HOTAIR is able to interact with the lysine-specific demethylase 1 (LSD1)-CoREST complex to mediate the removal of mono- and dimethylation from $\mathrm{H} 3 \mathrm{~K} 4$, a histone marker associated with gene activation $(6,8)$.

The results from current studies indicate that HOTAIR is a prognostic factor for the survival of patients with breast, colon cancer and glioma, and increased HOTAIR expression in patients has been correlated with increased metastasis (6,9-11). However, the expression and function of HOTAIR in TC is not well known. The present study demonstrated that the dysregulation of HOTAIR is correlated with metastasis and poor prognosis in patients with TC. Through loss-of-function analysis, the biological function of HOTAIR has been verified in human thyroid carcinoma cell lines. Collectively, the results of the present study demonstrate that HOTAIR may act as a novel biomarker in patients with TC.

\section{Materials and methods}

Tissue and plasma samples. Thyroid tissue samples from 35 patients with TC were collected via surgery from the Department of Thyroid Surgery and Gastrointestinal Surgery, at the Affiliated Yantai Yuhuangding Hospital of Qingdao University Medical College (Yantai, China) between September 
2012 and October 2014. Patients involved in the present study provided written informed consent. Adjacent tissue and cancerous tissue were reviewed and classified by a pathologist. Fresh tissue samples were frozen to liquid nitrogen within 30 min of surgery. Tissue sections from each TC sample.

Whole plasma samples were obtained from the 35 patients with TC and 20 healthy volunteers, and then stored in EDTA tubes (Zhongyuan Biotech, Beijing, China). The tubes were centrifuged at $1,200 \times \mathrm{g}$ for $10 \mathrm{~min}$ at $4^{\circ} \mathrm{C}$ to spin down the plasma cells. The supernatants were transferred to microcentrifuge tubes (Zhongyuan Biotech) and then centrifuged at $12,000 \times \mathrm{g}$ for $10 \mathrm{~min}$ at $4^{\circ} \mathrm{C}$ again to completely remove the cellular components. The plasma was then carefully collected, aliquoted, and stored at $-80^{\circ} \mathrm{C}$ until forthputting. Total RNA from $1 \mathrm{ml}$ plasma was extracted using TRIzol (Invitrogen; Thermo Fisher Scientific, Inc., Waltham, MA, USA) according to the manufacturer's instructions.

Reverse transcription quantitative-polymerase chain reaction (RT-qPCR). RT reactions were carried out in $1 \mu \mathrm{g}$ total RNA using the PrimeScript RT reagent kit (Takara Bio, Inc., Otsu, Japan). RT-qPCR was then performed using a SYBR Premix Dimer Eraser kit (Takara Bio, Inc.). 18S rRNA was evaluated as a housekeeping gene for the $\mathrm{qPCR}$ reactions. The primers used were as follows: HOTAIR forward, 5'-TCATGATGG AATTGGAGCCTT-3', and reverse, 5'-CTCTTCCTGGCT TGCAGATTG-3'; 18S rRNA forward, 5'-AGGATCCATTGG AGGGCAAGT-3', and reverse, 5'-TCCAACTACGAGCTT TTTAACTGCA-3'. All the reactions were carried out on an ABI7300 real-time PCR system according to the manufacturer's instructions. Cycling conditions were as follows: $95^{\circ} \mathrm{C}$ for $10 \mathrm{sec}$, one cycle; $95^{\circ} \mathrm{C}$ for $5 \mathrm{sec}, 60^{\circ} \mathrm{C}$ for $30 \mathrm{sec}, 40$ cycles; followed by a 30 -min melting curve collection to verify the primer dimers. The expression levels of HOTAIR in each sample were normalized to that of the internal control $18 \mathrm{~S}$ rRNA. The fold change of HOTAIR expression in the tissue samples and plasma samples compared with the controls were calculated using the $2^{-\Delta \Delta \mathrm{Ct}}$ method.

Cell lines and culture conditions. The HT-ori3 normal human thyroid cell line and human thyroid carcinoma cell lines including WRO, TPC-1 and SW579 were all purchased from Beijing Zhongyuan Ltd. (Beijing, China). All cells were maintained in a humidified atmosphere containing $10 \% \mathrm{CO}_{2}$ at $37^{\circ} \mathrm{C}$.

Small interfering (si)RNA transfection. Both HOTAIR siRNA and scramble were purchased from Qiagen (Hilden, Germany). Cells $\left(1 \times 10^{5}\right)$ were grown on six-well plates to $70 \%$ confluency and transfected using Lipofectamine ${ }^{\mathrm{TM}}$ RNAiMax (Invitrogen; Thermo Fisher Scientific, Inc.) according to the manufacturer's instructions. A total of $48 \mathrm{~h}$ post-transfection, the cells were harvested for RT-qPCR to analyze HOTAIR knockdown efficiency.

Cell proliferation assay. A cell counting kit-8 (CCK-8) cell proliferation kit was purchased from Dojindo Laboratories, (Kumamoto, Japan). All the experimental protocols were conducted in accordance with the manufacturers' instructions. Briefly, cells were seeded into a 96 -well plate at $1 \times 10^{3}$ cells/well
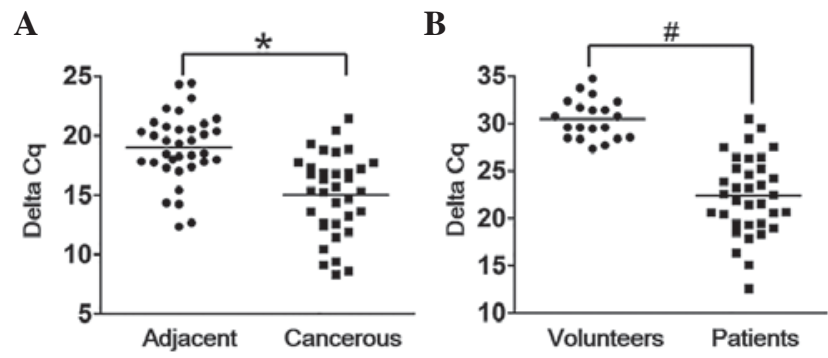

Figure 1. Expression of HOTAIR is elevated in TC tissues and plasma. (A) The expression levels of HOTAIR were detected in both adjacent tissues and cancerous tissues via reverse transcription-quantitative polymerase chain reaction. (B) The expression levels of plasma HOTAIR were analyzed in 35 patients with TC and 20 healthy volunteers. The experiments were repeated in triplicate and three independent experiments were performed. ${ }^{*} \mathrm{P}<0.05$; ${ }^{\mathrm{P}}<0.01$, vs. the control. HOTAIR, HOX transcript antisense RNA; $\mathrm{TC}$, thyroid cancer.

and cultured at $37^{\circ} \mathrm{C}$. CCK- 8 solution was added to each well at the indicated times points and then incubated at $37^{\circ} \mathrm{C}$ at 0 , 12, 24, 36 and $48 \mathrm{~h}$, then for a futher $2 \mathrm{~h}$. The absorbance at $450 \mathrm{~nm}$ was measured with a microplate reader (Bio-Rad Laboratories, Inc., Hercules, CA, USA). The experiments were repeated in triplicate and three independent experiments were performed.

Cell invasion assay. 24-well transwell plates (Corning Life Sciences, Tewksbury, MA, USA) were used for invasion assays. For in vitro invasion assays, the upper chambers of the transwells $(8 \mu \mathrm{m})$ were pre-coated with diluted matrigel (BD Biosciences, Franklin Lakes, NJ, USA). Briefly, $1 \times 10^{5}$ cells (in serum-free media) and $10 \%$ serum-containing media were plated in the upper chambers. After $48 \mathrm{hr}$ incubation, the invaded cells were stained with $0.1 \%$ crystal violet, and positively stained cells were counted with a microplate reader (Bio-Rad Laboratories, Inc.). The experiments were repeated in triplicate and three independent experiments were performed.

Statistical analysis. Quantitative variables were expressed as means \pm standard deviations in the statistical analysis. Statistical significances between groups were determined by two-tailed Student's $t$-test. All statistical analyses were carried out with SPSS 13.0 (SPSS, Inc., Chicago, IL, USA). P<0.05 was considered to indicate a statistically significant result. The survival calculations were illustrated with Kaplan-Meier curve.

\section{Results}

Expression levels of HOTAIR are elevated in TC tissue samples and plasma. To assess the potential biological function of HOTAIR, its expression levels in both adjacent tissues and cancerous tissues were analyzed by RT-qPCR. The results demonstrated that HOTAIR was significantly upregulated in cancerous tissues compared with adjacent tissues (Fig. 1A). The expression levels of plasma HOTAIR were also detected via RT-qPCR, and the results demonstrated that HOTAIR could be detected in TC patient plasma, whereas there was almost no HOTAIR expression in the plasma of the healthy volunteers (Fig. 1B). 
A

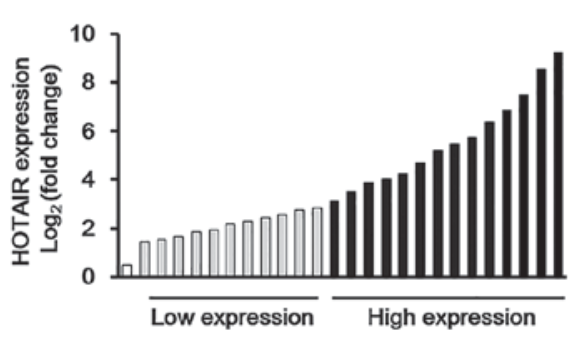

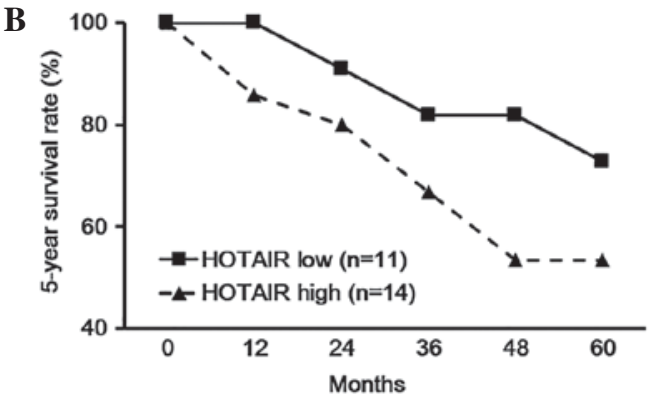

Figure 2. The association between plasma HOTAIR expression levels and 5-year survival rates. (A) The $35 \mathrm{TC}$ cases were divided into two groups according to the expression levels of plasma HOTAIR; 11 cases exhibited low HOTAIR expression levels and 14 cases exhibited high HOTAIR expression levels. (B) The association between plasma HOTAIR expression levels and 5-year survival rate was calculated with Kaplan-Meier analysis. HOTAIR, HOX transcript antisense RNA; TC, thyroid cancer.

A

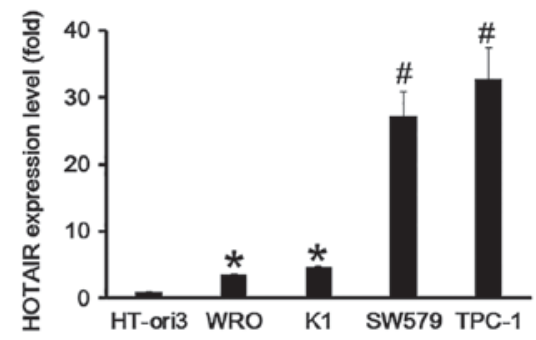

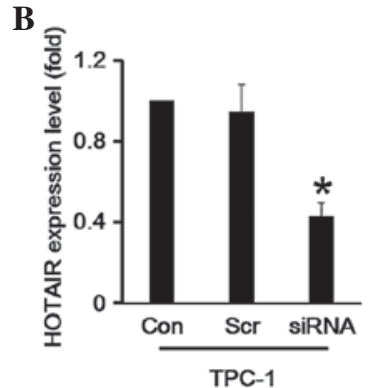

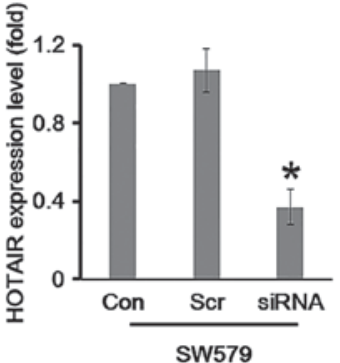

Figure 3. HOTAIR is upregulated in TC cell lines. (A) The expression levels of HOTAIR were detected in WRO, TPC-1 and SW579 cells via RT-qPCR, and HT-ori3 was used as the negative control. (B) The expression levels of HOTAIR were verified in TPC-1 and SW579 cell lines with a HOTAIR knockdown assay. Scr, cells transfected with scramble nucleotide fragments; siRNA, cells transfected with siRNA specifically targeting HOTAIR. The experiments were repeated in triplicate and three independent experiments were performed. ${ }^{\mathrm{P}}<0.05$; ${ }^{*} \mathrm{P}<0.01$, vs. the control. HOTAIR, HOX transcript antisense RNA; TC, thyroid cancer; siRNA, small interfering RNA; RT-qPCR, reverse transcription-quantitative polymerase chain reaction.

A

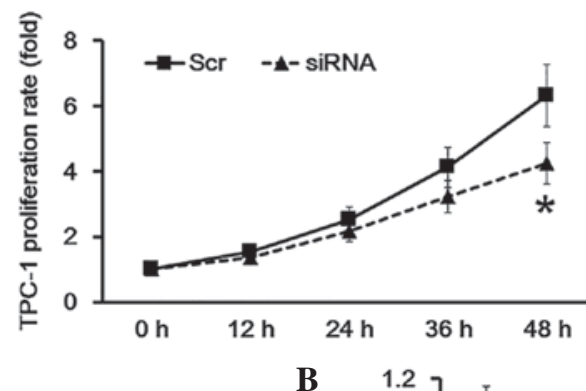

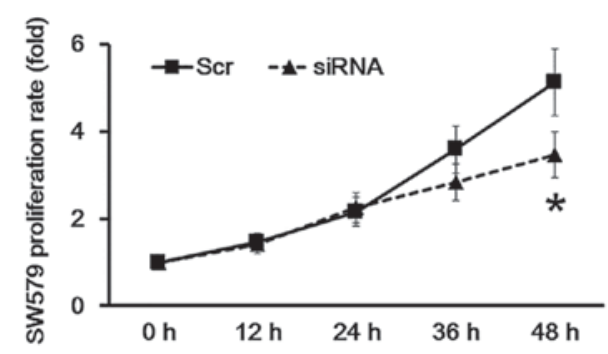

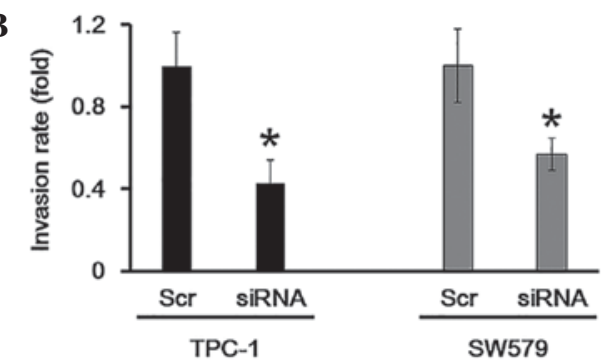

Figure 4. HOTAIR knockdown inhibits cell growth and invasion. (A) The cell proliferation rate was determined by cell counting kit-8 analysis in TPC-1 and SW579 cells in which HOTAIR was knocked down; cells transfected with scramble nucleotide fragment was used as the control. (B) The cell invasion ability was detected by transwell assay in TPC-1 and SW579 cells with HOTAIR knocked down; cells transfected with scramble nucleotide fragment was used as the control. The experiments were repeated in triplicate and three independent experiments were performed. "P $<0.05$, vs. the control. Scr, cells transfected with scramble nucleotide fragments; siRNA, cells transfected with siRNA specifically targeting HOTAIR. siRNA, small interfering RNA; HOTAIR, HOX transcript antisense RNA.

Association between plasma HOTAIR and 5-year survival rates. According to the expression levels of plasma HOTAIR obtained from the RT-qPCR assay, the 35 TC cases could be divided into two groups; one group that exhibited high HOTAIR expression levels, and the other low HOTAIR expression levels (Fig. 2A). Furthermore, the association between 
plasma HOTAIR expression levels and 5-year survival rate was analyzed, and the results demonstrated that higher expression levels of plasma HOTAIR were positively correlated with worse 5-year survival rates in patients with TC (Fig. 2B).

HOTAIR expression is upregulated in TC cell lines. The detected HOTAIR expression in TC patient plasma prompted further investigation into the function of HOTAIR. The HT-ori3 normal human thyroid cell line and three human thyroid carcinoma cell lines, including WRO, TPC-1 and SW579, were selected for further study. The results demonstrated that the expression of HOTAIR in human thyroid carcinoma cell lines showed an aberrant expression profile. The expression of HOTAIR was markedly upregulated in TPC-1 and SW579 cell lines $(\mathrm{P}<0.05)$, while HOTAIR was somewhat elevated in the WRO cell line compared with HT-ori3 ( $>>0.05)$, as determined by RT-qPCR (Fig. 3A). In order to explore the function of HOTAIR in TC cells, a siRNA specifically targeting HOTAIR was designed to knockdown endogenous HOTAIR. The results of the RT-qPCR demonstrated that HOTAIR was knocked down effectively in TPC-1 and SW579 cells (Fig. 3B).

HOTAIR knockdown inhibits cell growth and invasion. CCK-8 analysis was employed to determine the cell proliferation rate. The results demonstrated that when HOTAIR was knocked down in TPC-1 and SW579 cells, the proliferation was significantly inhibited compared with the scramble group (Fig. 4A). Additionally, a transwell assay was performed to study whether HOTAIR participated in cell invasion. The results suggested that HOTAIR knockdown in TPC-1 and SW579 cells resulted in a decreased invasion ability compared with the scramble group. These results indicated that HOTAIR knockdown suppressed cell proliferation and invasion.

\section{Discussion}

Significant progress in understanding the molecular pathogenesis of TC has been made in recent years. Remarkable knowledge has been accumulated on the role of fundamental signaling pathways, such as the mitogen-activated protein kinase and the phosphoinositide 3-kinase/protein kinase $\mathrm{B} /$ mammalian target of rapamycin pathways (4). Though these signaling pathways provide targets for therapeutic agents, a further and more detailed investigation of the molecular mechanism underlying TC tumorigenesis is required.

In recent years, studies on noncoding RNAs has attracted considerable attention in basic medical research $(12,13)$. It was reported that miRNAs and lncRNAs have distinct expression profiles in human plasma in various types of cancers (14). In addition, serum miRNAs may serve as important diagnostic biomarkers for certain cancer types, such as lung cancer (15), breast cancer (16), liver cancer (17) and pancreatic cancer (18). However, IncRNA expression patterns in plasma have not been investigated for their potential as novel biomarkers for TC diagnosis or prognosis.

LncRNAs, ranging from 200 to $>10,000$ nucleotides, are abundantly transcribed by the mammalian genome (19). LncRNAs have emerged as novel important regulators of tumorigenesis and development. For instance, the lncRNA
HOXA distal transcript antisense RNA is able to promote progression and gemcitabine resistance by regulating HOXA13 in pancreatic cancer (20); a novel lncRNA AK001796 may act as an oncogene and is involved in cell growth inhibition by resveratrol in lung cancer (21); lncRNA colon cancer associated transcript 1 (non-protein coding) is able to promote hepatocellular carcinoma progression by functioning as a let-7 sponge (22).

LncRNA HOTAIR was first discovered by Howard Chang's group (5), HOTAIR is overexpressed in breast, colorectal, liver and nasopharyngeal cancers (23). Tsai et al (8) reported that a 89 bp fragment at the 5 ' end of HOTAIR binds to PRC2, and a 646 bp fragment at its $3^{\prime}$ end binds to the LSD1/CoREST complex (8). HOTAIR has important roles in tumorigenesis and progression. Briefly, HOTAIR acts as an oncogene in tumorigenesis, and promotes invasion and metastases in tumor progression (24). However, whether HOTARI is involved in $\mathrm{TC}$ remains unclear. Therefore, the expression and function of HOTAIR must be comprehensively understood prior to the use of HOTAIR in TC treatment.

In the current study, the data suggested that HOTAIR is differentially expressed in the tissues and plasma of the patients with TC compared with the controls, and HOTAIR expression in TC is likely to be associated with the aggressiveness and the progression of the tumor. The in vitro experiments indicated that HOTAIR was able to act as an oncogene; knockdown of HOTAIR inhibited TC cancer cell proliferation and invasion. In conclusion, to the best of our knowledge, these findings indicate for the first time that the expression of HOTAIR in plasma can be used as a novel diagnostic biomarker for TC. The utility of HOTAIR expression could be established as a prognostic indicator for TC.

\section{References}

1. Fofi C, Festuccia F, Barberi S, Apponi F, Chiacchiararelli L, Scopinaro F, Punzo G and Menè P: Hemodialysis in patients requiring $131 \mathrm{I}$ treatment for thyroid carcinoma. Int $\mathbf{J}$ Artif Organs 36: 439-443, 2013.

2. Sherman EJ, Su YB, Lyall A, Schöder H, Fury MG, Ghossein RA, Haque S, Lisa D, Shaha AR, Tuttle RM and Pfister DG: Evaluation of romidepsin for clinical activity and radioactive iodine reuptake in radioactive iodine-refractory thyroid carcinoma. Thyroid 23: 593-599, 2013.

3. Wakabayashi H, Taki J, Inaki A, Toratani A, Kayano D and Kinuya S: Extremity Radioactive Iodine Uptake on Post-therapeutic Whole Body Scan in Patients with Differentiated Thyroid Cancer. Asia Ocean J Nucl Med Biol 3: 26-34, 2015.

4. Netea-Maier RT, Klück V, Plantinga TS and Smit JW: Autophagy in thyroid cancer: Present knowledge and future perspectives. Front Endocrinol (Lausanne) 6: 22, 2015.

5. Mattick JS: RNA regulation: A new genetics? Nat Rev Genet 5: 316-323, 2004.

6. Gupta RA, Shah N, Wang KC, Kim J, Horlings HM, Wong DJ, Tsai MC, Hung T, Argani P, Rinn JL, et al: Long non-coding RNA HOTAIR reprograms chromatin state to promote cancer metastasis. Nature 464: 1071-1076, 2010.

7. Khalil AM, Guttman M, Huarte M, Garber M, Raj A, Rivea Morales D, Thomas K, Presser A, Bernstein BE, van Oudenaarden A, et al: Many human large intergenic noncoding RNAs associate with chromatin-modifying complexes and affect gene expression. Proc Natl Acad Sci USA 106: 11667-11672, 2009.

8. Tsai MC, Manor O, Wan Y, Mosammaparast N, Wang JK, Lan F, Shi Y, Segal E and Chang HY: Long noncoding RNA as modular scaffold of histone modification complexes. Science 329: 689-693, 2010. 
9. Huang L, Liao LM, Liu AW, Wu JB, Cheng XL, Lin JX and Zheng M: Overexpression of long noncoding RNA HOTAIR predicts a poor prognosis in patients with cervical cancer. Arch Gynecol Obstet 290: 717-723, 2014.

10. Kogo R, Shimamura T, Mimori K, Kawahara K, Imoto S, Sudo T, Tanaka F, Shibata K, Suzuki A, Komune S, et al: Long noncoding RNA HOTAIR regulates polycomb-dependent chromatin modification and is associated with poor prognosis in colorectal cancers. Cancer Res 71: 6320-6326, 2011.

11. Zhang JX, Han L, Bao ZS, Wang YY, Chen LY, Yan W, Yu SZ, Pu PY, Liu N, You YP, et al: HOTAIR, a cell cycle-associated long noncoding RNA and a strong predictor of survival, is preferentially expressed in classical and mesenchymal glioma. Neuro Oncol 15: 1595-1603, 2013.

12. Thum T and CondorelliG: Long noncoding RNAs and microRNAs in cardiovascular pathophysiology. Circ Res 116: 751-762, 2015.

13. Ling H: Non-coding RNAs: Therapeutic Strategies and Delivery Systems. Adv Exp Med Biol 937: 229-237, 2016.

14. Latronico MV and Condorelli G: Therapeutic applications of noncoding RNAs. Curr Opin Cardiol 30: 213-221, 2015.

15. He D, Wang J, Zhang C, Shan B, Deng X, Li B, Zhou Y, Chen W, Hong J, Gao Y, et al: Down-regulation of miR-675-5p contributes to tumor progression and development by targeting pro-tumorigenic GPR55 in non-small cell lung cancer. Mol Cancer 14: 73, 2015.

16. Hu Y, Xu K and Yagüe E: MiR-218 targets survivin and regulates resistance to chemotherapeutics in breast cancer. Breast Cancer Res Treat 151: 269-280, 2015.
17. Rebucci M, Sermeus A, Leonard E, Delaive E, Dieu M, Fransolet M, Arnould T and Michiels C: MiRNA-196b inhibits cell proliferation and induces apoptosis in HepG2 cells by targeting IGF2BP1. Mol Cancer 14: 79, 2015.

18. Jin X, Sun Y, Yang H, Li J, Yu S, Chang X, Lu Z and Chen J: Deregulation of the MiR-193b-KRAS axis contributes to impaired cell growth in pancreatic cancer. PloS One 10: e0125515, 2015.

19. Kohls K, Schmidt D, Holdenrieder S, Müller SC and Ellinger J: Detection of cell-free lncRNA in serum of cancer patients. Urologe A 54: 819-825, 2015 (In German).

20. Li Z, Zhao X, Zhou Y, Liu Y, Zhou Q, Ye H, Wang Y, Zeng J, Song Y, Gao W, et al: The long non-coding RNA HOTTIP promotes progression and gemcitabine resistance by regulating HOXA13 in pancreatic cancer. J Transl Med 13: 84, 2015.

21. Yang Q, Xu E, Dai J, Liu B, Han Z, Wu J, Zhang S, Peng B, Zhang Y and Jiang Y: A novel long noncoding RNA AK001796 acts as an oncogene and is involved in cell growth inhibition by resveratrol in lung cancer. Toxicol Appl Pharmacol 285: 79-88, 2015.

22. Deng L, Yang SB, Xu FF and Zhang JH: Long noncoding RNA CCAT1 promotes hepatocellular carcinoma progression by functioning as let-7 sponge. J Exp Clin Cancer Res 34: 18, 2015.

23. Ishibashi M, Kogo R, Shibata K, Sawada G, Takahashi Y, Kurashige J, Akiyoshi S, Sasaki S, Iwaya T, Sudo T, et al: Clinical significance of the expression of long non-coding RNA HOTAIR in primary hepatocellular carcinoma. Oncol Rep 29: 946-950, 2013.

24. Yu X and Li Z: Long non-coding RNA HOTAIR: A novel oncogene (Review). Mol Med Rep 12: 5611-5618, 2015. 